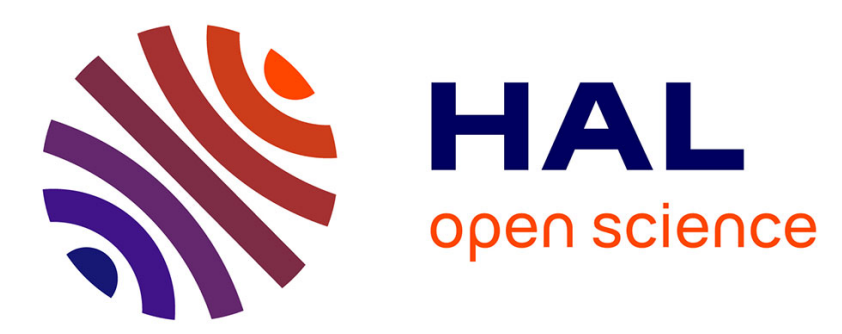

\title{
Feedforward control of open channel flow using differential flatness
}

T. Rabbani, Florent Di Meglio, X. Litrico, Aurélie Bayen-Poisson

\section{To cite this version:}

T. Rabbani, Florent Di Meglio, X. Litrico, Aurélie Bayen-Poisson. Feedforward control of open channel flow using differential flatness. IEEE Transactions on Control Systems Technology, 2010, 18 (1), p. 213 - p. 221. 10.1109/TCST.2009.2014640 . hal-00583606

\section{HAL Id: hal-00583606 https://hal.science/hal-00583606}

Submitted on 6 Apr 2011

HAL is a multi-disciplinary open access archive for the deposit and dissemination of scientific research documents, whether they are published or not. The documents may come from teaching and research institutions in France or abroad, or from public or private research centers.
L'archive ouverte pluridisciplinaire HAL, est destinée au dépôt et à la diffusion de documents scientifiques de niveau recherche, publiés ou non, émanant des établissements d'enseignement et de recherche français ou étrangers, des laboratoires publics ou privés. 


\title{
Feed-Forward Control of Open Channel Flow Using Differential Flatness
}

\author{
Tarek S. Rabbani, Florent Di Meglio, Xavier Litrico, and Alexandre M. Bayen
}

\begin{abstract}
This brief derives a method for open-loop control of open channel flow, based on the Hayami model, a parabolic partial differential equation resulting from a simplification of the SaintVenant equations. The open-loop control is represented as infinite series using differential flatness, for which convergence is assessed. A comparison is made with a similar problem available in the literature for thermal systems. Numerical simulations show the effectiveness of the approach by applying the open-loop controller to irrigation canals modeled by the full Saint-Venant equations.
\end{abstract}

Index Terms-Differential flatness, diffusive wave equation, open channel hydraulics, trajectory planning, water management.

\section{INTRODUCTION}

$\mathbf{T}$ HE limitation of global water resources is a motivation for research on automation of management of water distribution systems. Large amounts of fresh water are lost due to poor management of open-channel systems. This brief focuses on the management of canals used to convey water from the resource (generally a dam located upstream) to a specific downstream location. Due to the fluctuations of water needs, water demand changes with time. This change in demand calls for the efficient operations of open-channel systems to avoid overflows and to supply desired flow rates at prespecified time instants.

Automation techniques based on optimization and control have the potential to provide more efficient management strategies than manual techniques. They rely on flow models, in particular the Saint-Venant equations [1] or simplified versions of these equations to describe 1-D hydraulic systems. Water level regulation and control of the water flow are among the methods used to improve the efficiency of irrigation systems. These techniques allow engineers to regulate the flow in hydraulic canals and therefore to irrigate large areas according to user specified demands.

In this brief, the specific problem of controlling the downstream flow in a 1-D hydraulic canal by the upstream discharge is investigated. Several approaches to this problem have already been described in the literature. The majority of these approaches use linear controllers to control the (nonlinear)

Manuscript received April 13, 2008; revised September 27, 2008. Manuscript received in final form January 28, 2009. Recommended by Associate Editor B. de Jager. This work was supported by Cemagref and the France-Berkeley Fund.

T. Rabbani is with the Department of Mechanical Engineering, University of California, Berkeley, CA 94720 USA (e-mail: trabbani@ berkeley.edu).

F. Di Meglio is with the Ecole des Mines de Paris, 60 BD St-Mitchel, 75272 Paris Cedex 06, France.

X. Litrico was with the Civil and Environmental Engineering Department, University of California, Berkeley, CA 94720 USA. He is now with the UMR G-EAU, Cemagref, BP 5096, F-34196 Montpellier Cedex 5, France.

A. Bayen is with the Systems Engineering Program, Department of Civil and Environmental Engineering, University of California, Berkeley, CA 94720 USA.

Color versions of one or more of the figures in this brief are available online at http://ieeexplore.ieee.org.

Digital Object Identifier 10.1109/TCST.2009.2014640 dynamics of the canal system. Such methods include transfer function analysis for Saint-Venant equations [19] which in turn allows the use of classical control techniques for feedback control [6], [20]. Alternatively, Riemann invariants for hyperbolic conservation laws as in [7] and [8] can be used to construct Lyapunov functions, used for stabilization purposes. Adjoint methods [32] have been used for estimation and control, via sensitivity analysis. More closely related to the present study, open-loop control methods have been developed either by computing the solutions of the flow equations backwards using discretization and finite difference methods [3], [4], or using a finite dimensional approximation in the frequency domain [21], [28]. Our approach is to design an open-loop controller which parametrizes the upstream discharge explicitly as a function of the desired downstream discharge at a given location using differential flatness (based on Cauchy-Kovalevskaya series). It can be shown using Lyapunov stability method that the open-loop system is stable [15], [16], which provides another justification for the usefulness of open-loop control of the considered system.

In the context of partial differential equations, differential flatness was used to investigate the related problem of heavy chains motion planning [27], as well as Burgers equation in [26] or the telegraph equation in [12]. The theory of differential flatness, which was first developed in [11], consists in a parametrization of the trajectories of a system by one of its outputs, called the "flat output".

Starting from the classical Saint-Venant equations, widely used, to model unsteady flows in rivers [1], we present a model simplification and a linearization which lead to the Hayami partial differential equation as shown in [25]. The practicality of using the Hayami equation lies in the fact that only two numerical parameters are needed to characterize flow conditions: celerity and diffusivity. The original Saint-Venant equations require the knowledge of the full geometry of the canal and of the roughness coefficient, which make it impractical for long rivers where these parameters are more difficult to infer [18].

The problem of controlling the Hayami equation was already investigated [22] with transfer function analysis, and in [18] for parameter estimation. The Hayami equation [14] is closely linked to the diffusive wave equation with quadratic source terms, which has been studied in [10] and [23]. The difference between our problem and the aforementioned problem is the nature of the boundary conditions: indeed, unlike for heat transfer problems, one cannot impose a value for the downstream discharge (respectively, heat flux). In river flow, there are hydraulic structures such as weirs or gates which impose a static relation between water elevation and the flow. In fact, we show that the solution of our problem is a composite of the solution in [23] and an additional new term which captures the boundary condition set by the hydraulic structure, therefore required to solve the specific problem of interest in this study. 
This brief is organized as follows. A description of the physical problem and the system of equations to be solved is first introduced (see Section II). Then, in Section III, a solution of these equations is derived using differential flatness. The convergence of the infinite series controller is studied and an upper bound on the truncation error is computed as a function of the approximating terms. Moreover, a numerical assessment of the openloop controller is finally presented and discussed in Section IV. In particular, the difference with controllers synthesized in the context of heat transfer is illustrated through numerical simulation. Applications of the controller on the fully nonlinear Saint-Venant model are presented to show the usefulness of the proposed method for the full nonlinear system.

\section{PhysicAl Problem}

The system of interest is a hydraulic canal of length $L$. For simplicity, the canal is assumed to have a uniform rectangular cross-section but more complex geometries can easily be taken into account. In this section, we present the equations that govern the system, the Saint-Venant equations. We then derive the Hayami model which is a simplification of these equations.

\section{A. Saint-Venant Equations}

The Saint-Venant equations [1] are generally used to describe unsteady flows in rivers or canals [25]. These equations assume 1-D flow, with uniform velocity over the cross-section. The effect of boundary friction and turbulence is accounted for through resistance laws such as the Manning-Strickler formula [33], the average channel bed slope is assumed to be small, and the pressure is hydrostatic. Under these assumptions, these equations are written as follows:

$$
\begin{aligned}
A_{t}+Q_{x} & =0 \\
Q_{t}+\left(\frac{Q^{2}}{A}\right)_{x}+g A\left(Y_{d}\right)_{x} & =g A\left(S_{b}-S_{f}\right)
\end{aligned}
$$

with $A(x, t)$ the wetted cross-sectional area $\left(\mathrm{m}^{2}\right), Q(x, t)$ the discharge $\left(\mathrm{m}^{3} / \mathrm{s}\right)$ across section $A(x, t), Y_{d}(x, t)$ the water depth $(\mathrm{m}), S_{f}(x, t)$ the friction slope $(\mathrm{m} / \mathrm{m}), S_{b}$ the bed slope $(\mathrm{m} / \mathrm{m})$, and $g$ the gravitational acceleration $\left(\mathrm{m}^{2} / \mathrm{s}\right)$. For rectangular cross sectional geometries, these variables are linked by the following relations: $A(x, t)=Y_{d}(x, t) B_{0}$, $Z(x, t)=Y_{d}(x, t)+S_{b}(L-x)$ and $Q(x, t)=V(x, t) A(x, t)$ where $Z(x, t)$ is the absolute water elevation $(\mathrm{m}), V(x, t)$ is the mean water velocity $(\mathrm{m} / \mathrm{s})$ across section $A(x, t)$, and $B_{0}$ is the bed width (m). Equation (1) is referred to as the mass conservation equation, and (2) is called the momentum conservation equation. We assume that there is a cross-structure at the downstream end of the canal, which can be modeled by a static relation between $Q$ and $Z$ at $x=L$, i.e.,

$$
Q(L, t)=W(Z(L, t))
$$

where $W(\cdot)$ is an analytical function. For a weir structure, this relation can be assumed to be $Q(L, t)=$
$C_{w} \sqrt{2 g} L_{w}\left(Z(L, t)-Z_{w}\right)^{3 / 2}$, where $g$ is the gravitational acceleration, $L_{w}$ is the weir length, $Z_{w}$ is the weir elevation, and $C_{w}$ is the weir discharge coefficient. A similar static relation holds in the case of a gate structure.

\section{B. Hayami Model}

Depending on the characteristics of the river, some terms in the momentum equation (2) can be neglected, which allows us to simplify the two equations and to assemble them into a single partial differential equation. As shown in [22], assuming that the inertia terms $Q_{t}+\left(Q^{2} / A\right)_{x}$ can be neglected with respect to $g A\left(Y_{d}\right)_{x}$ will lead to the diffusive wave model

$$
\begin{aligned}
B_{0}\left(Y_{d}\right)_{t}+Q_{x} & =0 \\
Z_{x} & =-S_{f} .
\end{aligned}
$$

The two equations can be combined and will lead to the diffusive wave equation [18]

$$
Q_{t}+C Q_{x}-D Q_{x x}=0
$$

where $Q(x, t)$ is the flow $\left(\mathrm{m}^{3} / \mathrm{s}\right), C$ and $D$ usually known as the celerity and the diffusivity are non linear functions of the flow. Linearizing (4) around a reference discharge $Q_{0}$ (i.e., $Q(x, t)=$ $\left.Q_{0}+q(x, t)\right)$ leads to the Hayami equation

$$
q_{t}+C_{0} q_{x}-D_{0} q_{x x}=0
$$

where $q(x, t)$ is the deviation from the nominal flow $Q_{0}$, $C_{0}\left(Q_{0}\right)$, and $D_{0}\left(Q_{0}\right)$ are the nominal celerity and diffusivity which depend on $Q_{0}$. We call $Z_{0}$ the reference elevation, and assume that $Z(x, t)=Z_{0}+z(x, t)$, therefore (4) can be linearized as follows:

$$
B_{0} z_{t}+q_{x}=0
$$

where we have substituted $\left(Y_{d}\right)_{t}$ by $\left(Z-S_{b}(L-x)\right)_{t}=Z_{t}$ before linearizing. The right boundary condition (3) is also linearized and becomes

$$
q(L, t)=b z(L, t)
$$

where $b$ is the linearization constant $\left(\mathrm{m}^{2} / \mathrm{s}\right)$. The value of this constant depends on the weir geometry: length, height, and discharge coefficient.

\section{Open-Loop Control Problem}

The control problem illustrated in Fig. 1, consists in determining the control $u(t)=q(0, t)$, i.e., the flow of the upstream discharge that yields the desired downstream discharge $y(t)=q(L, t)$, where $y(t)$ is a user-defined flow profile over time at the end of the canal.

We therefore have to solve a feed-forward control problem for a system with boundary control (in the present case upstream discharge). The dynamics are modeled by the following partial differential equations: 


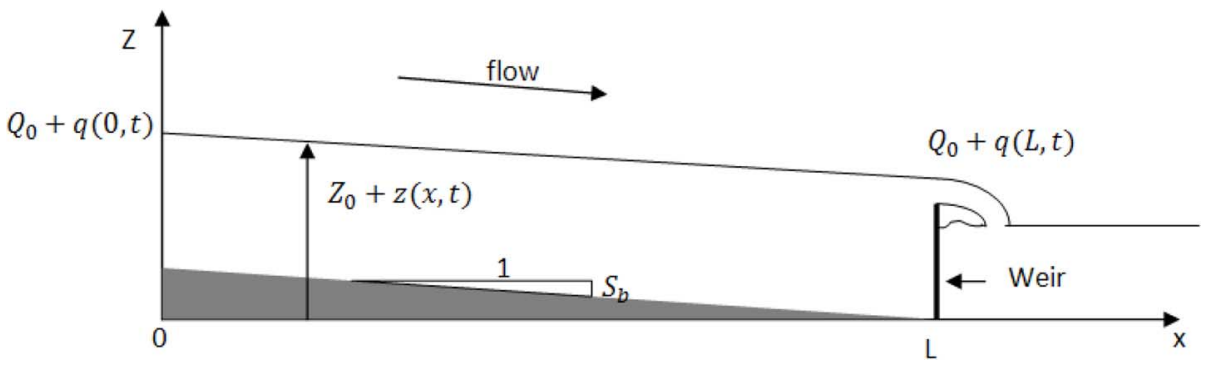

Fig. 1. Schematic representation of the canal with weir structure.

$$
\begin{array}{ll}
\forall x \in] 0, L[\forall t \in] 0, T] & D_{0} q_{x x}-C_{0} q_{x}=q_{t} \\
\forall x \in] 0, L[\forall t \in] 0, T] & B_{0} z_{t}+q_{x}=0 .
\end{array}
$$

A boundary condition is imposed at $x=L$ by (9)

$$
\forall t \in] 0, T] \quad q(L, t)=b z(L, t)=y(t)
$$

where $y(t)$ is the desired output, and initial conditions defined by the deviations from the nominal values

$$
\begin{aligned}
& \forall x \in] 0, L[\quad q(x, 0)=0 \\
& \forall x \in] 0, L[\quad z(x, 0)=0 .
\end{aligned}
$$

We are looking for the appropriate control $u(\cdot)$ that will generate the $y(t)$ defined by $(9)$, where $u(\cdot)$ is defined by

$$
\forall t \in] 0, T] \quad u(t)=q(0, t) .
$$

\section{COMPUTATION OF THE OPEN-LOOP CONTROL INPUT FOR THE HAYAMI MODEL}

In this section, we solve the control problem given by (7)-(9) and parametrize the flow $q(x, t)$ in terms of the discharge $q(L, t)$ or $y(t)$. We will produce a solution to this problem using differential flatness based on Cauchy-Kovalevskaya decomposition, and study the convergence of the obtained infinite series.

\section{A. Cauchy-Kovalevskaya Decomposition}

Following [29], (7) can be transformed into the heat equation. Let us consider the following transformation:

$$
q(x, t)=h(x, t) p(x, t)
$$

where $h(x, t)=e^{\left(-\left(\alpha^{2}\right) /\left(\beta^{2}\right) t+\alpha(x-L)\right)}, \alpha=\left(C_{0}\right) /\left(2 D_{0}\right)$, and $\left.\beta=(1) /\left(\sqrt{D_{0}}\right)\right)$. We have

$$
\begin{aligned}
p_{t} h(x, t) & =q_{t}+\frac{\alpha^{2}}{\beta^{2}} q \\
p_{x} h(x, t) & =q_{x}-\alpha q \\
p_{x x} h(x, t) & =q_{x x}-2 \alpha q_{x}+\alpha^{2} q .
\end{aligned}
$$

Substituting in (7), $p(x, t)$ satisfies

$$
p_{t}=\frac{1}{\beta^{2}} p_{x x} .
$$

The problem (7)-(9) can now be reformulated as follows:

$$
\begin{array}{ll}
\forall x \in] 0, L[\forall t \in] 0, T] & p_{t}=\frac{1}{\beta^{2}} p_{x x} \\
\forall x \in] 0, L[\forall t \in] 0, T] & B_{0} z_{t}=-h(x, t)\left(p_{x}+\alpha p\right) \\
\forall t \in] 0, T] & p(L, t)=f(t) y(t)
\end{array}
$$

where $f(t)=e^{\left(\alpha^{2} / \beta^{2}\right) t}$. The system of (13)-(15) can be used for a Cauchy-Kovalevskaya decomposition [5], [17] and the solution of the PDE, $p(x, t)$ (respectively, $z(x, t)$ ), can be expressed in the Cauchy-Kovalevskaya power series decomposition, in the present case as a function of $p(L, t)$ (respectively, $z(L, t))$ and all its derivatives. The Cauchy-Kovalevskaya decomposition is a standard way of parametrizing the input as a function of the output for parabolic and linear PDEs [10], [17], [23]. In the present case, it can be shown to be equivalent to Laplace decomposition [9] which produces the same parametrization, using spectral analysis. We assume the following form for $p$ and $z$ :

$$
\begin{aligned}
& p(x, t)=\sum_{i=0}^{+\infty} p_{i}(t) \frac{(x-L)^{i}}{i !} \\
& z(x, t)=\sum_{i=0}^{+\infty} z_{i}(t) \frac{(x-L)^{i}}{i !}
\end{aligned}
$$

where $p_{i}(t)$ and $z_{i}(t)$ are $C^{\infty}$ functions. We have: $p_{t}=$ $\sum_{i=0}^{+\infty} \dot{p}_{i}\left((x-L)^{i} / i !\right), p_{x x}=\sum_{i=0}^{+\infty} p_{i+2}\left((x-L)^{i} / i !\right)$, where $\dot{p}_{i}$ denotes the time derivative of $p_{i}(t)$. After substitution in (13), we obtain

$$
\sum_{i=0}^{+\infty} \dot{p}_{i} \frac{(x-L)^{i}}{i !}=\frac{1}{\beta^{2}} \sum_{i=0}^{+\infty} p_{i+2} \frac{(x-L)^{i}}{i !} .
$$

Equating the coefficients of $(x-L)^{i} / i$ ! gives for all $i \in \mathbb{N}$

$$
p_{i+2}(t)=\beta^{2} \dot{p}_{i}(t) .
$$

Additionally, it follows from (16) and (17) that $p_{0}=p(L, t)$ and $z_{0}=z(L, t)$. We still need a condition on $p_{1}$ to be able to express every $p_{i}$ as a function of $p_{0}$. We combine (14) and (15) to obtain a boundary condition on $p$ at $x=L$. We have

$$
z_{t}=\sum_{i=0}^{+\infty} \dot{z}_{i} \frac{(x-L)^{i}}{i !} .
$$

So that $\dot{z}_{0}=z_{t}(L, t)$, and (14), with $x=L$ gives

$$
B_{0} \dot{z}_{0}+e^{-\left(\alpha^{2} / \beta^{2}\right) t}\left(p_{1}+\alpha p_{0}\right)=0 .
$$


In addition, (15) gives: $p_{0}=b z_{0} e^{\left(\alpha^{2} / \beta^{2}\right) t}$. Differentiating this equation with respect to time, we get

$$
\dot{z}_{0}=\frac{1}{b}\left(\dot{p}_{0}-\frac{\alpha^{2}}{\beta^{2}} p_{0}\right) e^{-\left(\alpha^{2} / \beta^{2}\right) t}
$$

and eventually; plugging back into (19), we obtain: $p_{1}=-\left(B_{0} / b\right) \dot{p_{0}}+\kappa p_{0}$, where $\kappa=\left(B_{0} / b\right)\left(\alpha^{2} / \beta^{2}\right)-\alpha$. Using the induction relation (18) and the expression of $p_{0}$ and $p_{1}$, we can compute separately the odd and even terms

$$
\begin{aligned}
p_{2 i} & =\beta^{2 i} p_{0}^{(i)} \\
p_{2 i+1} & =\kappa \beta^{2 i} p_{0}^{(i)}-\frac{B_{0}}{b} \beta^{2 i} p_{0}^{(i+1)}
\end{aligned}
$$

where $p_{0}^{(i)}$ stands for the $i^{t h}$ time derivative of $p_{0}(t)$. Therefore, we can formally write $p(x, t)$ as follows:

$$
\begin{aligned}
p(x, t)= & \sum_{i=0}^{+\infty} \beta^{2 i} p_{0}^{(i)} \frac{(x-L)^{2 i}}{(2 i) !} \\
& +\sum_{i=0}^{+\infty} \beta^{2 i}\left(\kappa p_{0}^{(i)}-\frac{B_{0}}{b} p_{0}^{(i+1)}\right) \frac{(x-L)^{2 i+1}}{(2 i+1) !} .
\end{aligned}
$$

From (15), we deduce that $p_{0}(t)=f(t) y(t)$. The final parametrization of the flow $q(x, t)$ will have the form

$$
q(x, t)=h(x, t)\left(T_{1}(x, t)+\kappa T_{2}(x, t)-\frac{B_{0}}{b} T_{3}(x, t)\right)
$$

where

$$
\begin{aligned}
& T_{1}(x, t)=\sum_{i=0}^{+\infty}(f y)^{(i)} \frac{\beta^{2 i}(x-L)^{2 i}}{(2 i) !} \\
& T_{2}(x, t)=\sum_{i=0}^{+\infty}(f y)^{(i)} \frac{\beta^{2 i}(x-L)^{2 i+1}}{(2 i+1) !} \\
& T_{3}(x, t)=\sum_{i=0}^{+\infty}(f y)^{(i+1)} \frac{\beta^{2 i}(x-L)^{2 i+1}}{(2 i+1) !} .
\end{aligned}
$$

Equation (20) relates the discharge variation $q(x, t)$ as a function of the desired flat output $y(t)$ which corresponds to the discharge $q(L, t)$ at the downstream end of the canal. The output $y(t)$ is sometimes referred to as "flat," which in the present context means that it is possible to express the input of the system $u(t)$ explicitly as a function of the desired output $y(t)$ and its derivatives (a formal definition of differential flatness is available in [11], for general systems). This also defines the parametrization of the state $q(x, t)$ as a function of the same derivatives. The present decomposition, chosen for this study, is the Cauchy-Kovalevskaya form, which is appropriate for parabolic equations such as the one presented in this brief. This solution is formal, until the convergence of the infinite series is assessed. An alternate derivation of (20) was produced using Laplace techniques, and provides the same algebraic result [9].

\section{B. Convergence of the Infinite Series}

We now give the formal proof of convergence of the series in (20). We assume that the flat output $y(t)$ is a Gevrey function [30] of order $\gamma>0$, i.e.,

$$
\exists m, l>0 \quad \forall n \in \mathbb{N} \quad \sup _{t \in \mathbb{R}}\left|y^{(n)}(t)\right|<m \frac{(n !)^{\gamma}}{l^{n}} .
$$

$f(t)=e^{\left(\alpha^{2} / \beta^{2}\right) t}$ is Gevrey of order 0 , and therefore is Gevrey of order $\gamma$. The product of two Gevrey functions of same order is a Gevrey function of the same order, as a consequence, $f(t) y(t)$ is Gevrey of order $\gamma>0$. We will use the Cauchy-Hadamard theorem [13] which states that the radius of convergence of the Taylor series $\sum_{i=0}^{+\infty} a_{n} x^{n}$ is $\left(1 / \lim \sup _{n \rightarrow+\infty}\left|a_{n}\right|^{1 / n}\right)$. The radius of convergence for $T_{3}(x, t)$ is given by

$$
\frac{1}{\rho}=\limsup _{i \rightarrow+\infty}\left(\frac{\beta^{2 i}\left|(f y)^{(i+1)}(t)\right|}{(2 i+1) !}\right)^{(1 / 2 i+1)}
$$

where $\rho$ is the radius of convergence around $L$. We can find an upper bound to $(1 / \rho)$ by inducing the property of bounds on a Gevrey function of order $\gamma>0$ from (24)

$$
\begin{aligned}
\frac{1}{\rho} & \leq \limsup _{i \rightarrow+\infty}\left(\frac{\beta^{2 i} m \frac{((i+1) !)^{\gamma}}{l^{i+1}}}{(2 i+1) !}\right)^{\left(\frac{1}{2 i+1}\right)} \\
& \leq \limsup _{i \rightarrow+\infty} \frac{\beta^{\left(\frac{2 i}{2 i+1}\right)} m^{\left(\frac{1}{2 i+1}\right)}}{l^{\left(\frac{i+1}{2 i+1}\right)}\left(\frac{((i+1) !)^{\gamma}}{(2 i+1) !}\right)^{\left(\frac{1}{2 i+1}\right)}} \\
& \sim \limsup _{i \rightarrow+\infty} \frac{\beta}{\sqrt{l}} \frac{i+1}{2 i+1}\left(\frac{i+1}{e}\right)^{\frac{(\gamma-2) i+(\gamma-1)}{2 i+1}} \\
& \sim \begin{cases}+\infty & \gamma>2 \\
\frac{\beta}{2 \sqrt{l}} & \gamma=2 \\
0 & \gamma<2\end{cases}
\end{aligned}
$$

where in (25), we have used the fact that $((i+1) !)^{(1) /(i+1)} \sim$ $(i+1) /(e)$, and $((2 i+1) !)^{(1) /(2 i+1)} \sim(2 i+1) /(e)$ as an immediate consequence of the Stirling formula. Also, we have used $m^{(1) /(2 i+1)} \sim 1, \beta^{(2 i) /(2 i+1)} \sim \beta$ and $l^{(i+1) /(2 i+1)} \sim$ $\sqrt{l}$. This will ensure an infinite radius of convergence for $\gamma<$ 2. Similar calculations can be held for $T_{1}(x, t)$ and $T_{2}(x, t)$ leading to the following conclusions.

- Equation (20) converges with an infinite radius of convergence for the choice of a Gevrey function $y(t)$ of order $\gamma<2$.

- For $\gamma=2$, the radius of convergence is greater than $(2 \sqrt{l} / \beta)$, which provides convergence of the series for $x \in[L-(2 \sqrt{l} / \beta), L]$, given the definition of $x \in[0, L]$.

- We can draw no conclusions on the convergence of the series when $\gamma>2$.

\section{Numerical Assessment of the Performance of the FEED-FORWARD CONTROLLER}

In this section, we compute the control command $u(t)$ by evaluating (20) at $x=0$. We subsequently simulate the controller numerically on the Hayami model (7)-(9) in order to evaluate their behavior before testing them on the Saint-Venant equations. This section successively investigates numerical simulations for the Hayami and the Saint-Venant models and the performance of the controller on both models. 


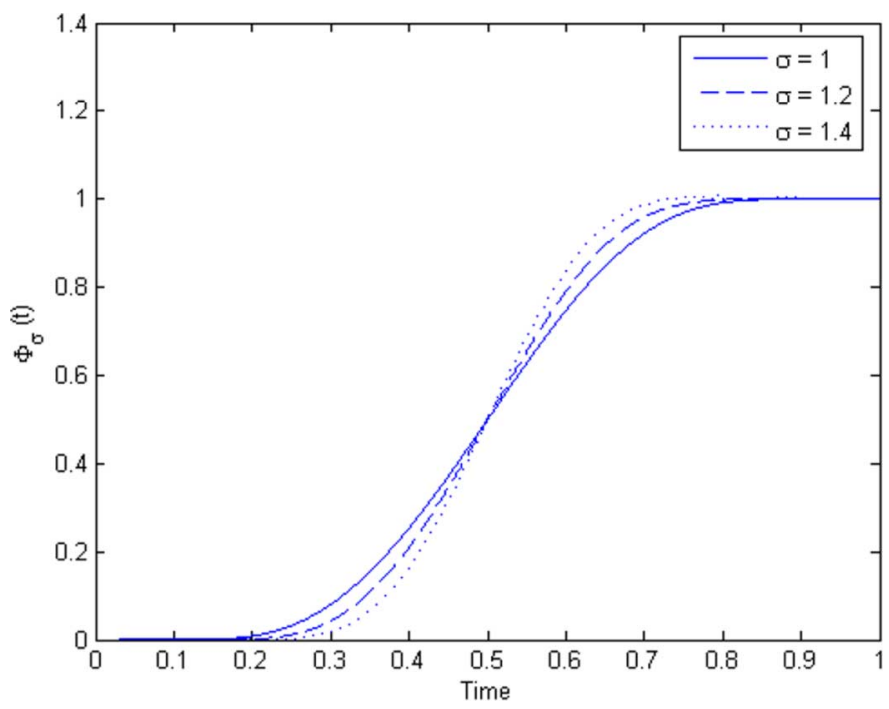

Fig. 2. Bump function described by (26) plotted for different values of $\sigma$ and $T=1$.

\section{A. Hayami Model Simulation}

From Section III-B, the infinite series convergence is ensured by choosing $y(t)$ to be a Gevrey function of order $\gamma<2$. To meet this convergence condition following [17], we introduce the bump function $\phi_{\sigma}(t): \mathbb{R} \rightarrow \mathbb{R}$ defined as

$$
\phi_{\sigma}(t)= \begin{cases}0, & t<0 \\ \frac{\int_{0}^{t / T} \exp \left(\frac{-1}{\left((\tau(1-\tau))^{\sigma}\right.}\right) d \tau}{\int_{0}^{1} \exp \left(\frac{-1}{\left((\tau(1-\tau))^{\sigma}\right.}\right) d \tau}, & 0 \leq t \leq T \\ 1, & t>T\end{cases}
$$

where $\sigma>1, T>0$. The Gevrey order of the bump function is $1+1 / \sigma$. The function $\phi_{\sigma}(t)$ is used in [10], [11], [17], [23], and [31], it is strictly increasing from 0 at $t=0$ to 1 at $t=T$ with zero derivatives at $t=0$ and $t=T$. The larger the $\sigma$ parameter is, the faster is the slope of transition. Fig. 2 shows a plot of the bump function for different values of $\sigma$ and $T=1$. Setting $y(t)=q_{1} \phi_{\sigma}(t)$ will allow us to have a transition from zero discharge flow for $t \leq 0$ to a discharge flow equal to $q_{1}$ for $t \geq T$, where $q_{1}$ is a constant. Note that the bump function was chosen because of its Gevrey properties, we guarantee an infinite radius of convergence for $\sigma>1$ ( $\gamma<2$ as described in Section III-B). As can be inferred from the previous proof, the proposed method only applies to functions with proper radius of convergence, by (25). This is due to the fact that in general, the reachable set (i.e., the set of attainable $y(\cdot)$ functions) from input functions $u(\cdot)$ is not equal to the whole state space of output functions. In other words, not all functions $y(\cdot)$ can be synthesized by a function $u(\cdot)$.

The upstream discharge or the control input $u(t)$ can be computed by substituting $x=0$ in (20). We obtain

$$
u(t)=h(0, t)\left(T_{1}(0, t)+\kappa T_{2}(0, t)-\frac{B_{0}}{b} T_{3}(0, t)\right) .
$$

1) Evaluation of the Truncation Error: For practical implementation purposes, one needs to know how many terms should be included in the numerical computation. This can be done by computing an upper bound on the truncation error. When the infinite series, $T_{1}(0, t), T_{2}(0, t)$, and $T_{3}(0, t)$, in (27) are truncated, this generates an approximation error which needs to be evaluated. We use the Gevrey assumption in (24) and write

$$
\begin{aligned}
\left|T_{1}(0, t)\right| \leq \sum_{i=0}^{\infty} b_{i}, \quad b_{i} & =m \frac{(i !)^{\gamma}}{l^{i}} \frac{[\beta L]^{2 i}}{(2 i) !}, \\
\left|T_{2}(0, t)\right| & \leq \sum_{i=0}^{\infty} c_{i}, \quad c_{i}=m \frac{(i !)^{\gamma}}{l^{i}} \frac{\beta^{2 i} L^{2 i+1}}{(2 i+1) !} \\
\left|T_{3}(0, t)\right| & \leq \sum_{i=0}^{\infty} d_{i}, \quad d_{i}=m \frac{((i+1) !)^{\gamma}}{l^{i+1}} \frac{\beta^{2 i} L^{2 i+1}}{(2 i+1) !} .
\end{aligned}
$$

To evaluate the approximation error of $T_{1}(0, t)$ when truncated, we study the series $b_{i}$. The series $b_{i}$ satisfies the relation $b_{i+1}=\mathcal{E}_{1}(i) b_{i}$, where $\mathcal{E}_{1}(i)=\left((i+1)^{\gamma}\right) /((2 i+2)(2 i+1))\left(\beta^{2} L^{2}\right) /(l)$. The function $\mathcal{E}_{1}(i)$ is decreasing towards zero

$$
\begin{aligned}
\frac{d\left(\mathcal{E}_{1}(i)\right)}{d i} & =\frac{(1+i)^{\gamma}(\gamma-3+2 i(\gamma-2))}{2\left(1+3 i+2 i^{2}\right)^{2}} \frac{\beta^{2} L^{2}}{l}<0 \\
\forall \gamma & <2
\end{aligned}
$$

and $\mathcal{E}_{1}(i) \sim\left(\beta^{2} L^{2}\right) /(4 l) i^{\gamma-2}$ for large values of $i$. Thus, for $\gamma<2$, this implies that, for any small enough constant $\epsilon<$ 1 , there exists a unique integer $i_{1}$ such that $\mathcal{E}_{1}\left(i_{1}\right) \leq \epsilon$ and $\mathcal{E}_{1}\left(i_{1}-1\right)>\epsilon$. Since $\mathcal{E}_{1}(i)$ is strictly decreasing, we have $\mathcal{E}_{1}(j) \leq \mathcal{E}_{1}\left(i_{1}\right) \leq \epsilon$ for any $j \geq i_{1}$. Thus, $b_{j+1} \leq b_{j} \epsilon$ and $b_{j+k} \leq b_{j} \epsilon^{k} \forall j \geq i_{1}, \forall k \geq 0 . T_{2}(0, t)$, and $T_{3}(0, t)$ satisfy similar properties, which can be summarized by: for any $\epsilon<1$, there exist $j \geq 0$, such that

$$
b_{j+k} \leq b_{j} \epsilon^{k}, \quad c_{j+k} \leq c_{j} \epsilon^{k}, \quad d_{j+k} \leq d_{j} \epsilon^{k} \forall k \geq 0
$$

This result provides us with an upper bound on the truncation error, which is quantified by writing (27) as a sum of the truncated series and the truncation error

$$
u(t)=u_{j}(t)+e_{j}(t)
$$

where

$$
\begin{aligned}
& u_{j}(t)=\left(\sum_{i=0}^{j-1}(f y)^{(i)} \frac{\beta^{2 i} L^{2 i}}{(2 i) !}-\kappa \sum_{i=0}^{j-1}(f y)^{(i)} \frac{\beta^{2 i} L^{2 i+1}}{(2 i+1) !}\right. \\
& \left.+\frac{B_{0}}{b} \sum_{i=0}^{j-1}(f y)^{(i+1)} \frac{\beta^{2 i} L^{2 i+1}}{(2 i+1) !}\right) \\
& e_{j}(t)=h(0, t)\left(\sum_{i=j}^{+\infty}(f y)^{(i)} \frac{\beta^{2 i} L^{2 i}}{(2 i) !}-\kappa \sum_{i=j}^{+\infty}(f y)^{(i)} \frac{\beta^{2 i} L^{2 i+1}}{(2 i+1) !}\right. \\
& \left.+\frac{B_{0}}{b} \sum_{i=j}^{+\infty}(f y)^{(i+1)} \frac{\beta^{2 i} L^{2 i+1}}{(2 i+1) !}\right) .
\end{aligned}
$$

We now use the geometric series upper bound given by (28) to compute an upper bound of the truncation error, for a large enough $j$

$$
\begin{aligned}
\left|u(t)-u_{j}(t)\right| & =\left|e_{j}(t)\right| \\
& \leq h(0, t)\left(b_{j} \sum_{k=0}^{\infty} \epsilon^{k}+|\kappa| c_{j} \sum_{k=0}^{\infty} \epsilon^{k}\right.
\end{aligned}
$$




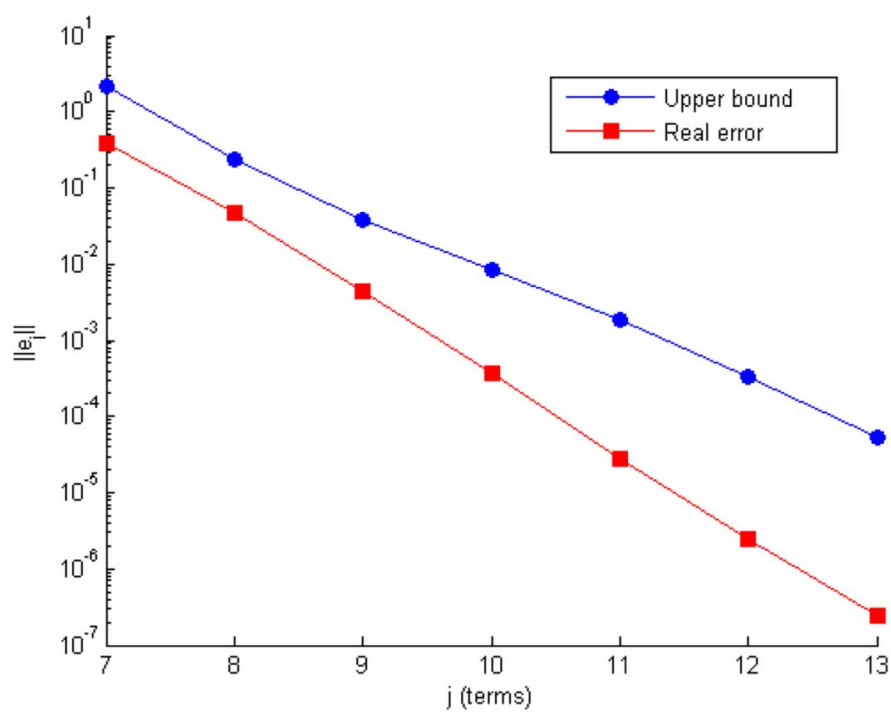

Fig. 3. $L_{2}$ norm of the error $e_{j}(t)$ defined by (31) as a function of the terms used $j$. The upper bound is computed using (32) and the real error is computed until numerical convergence.

$$
\begin{gathered}
\left.+\frac{B_{0}}{b} d_{j} \sum_{k=0}^{\infty} \epsilon^{k}\right) \\
\leq \frac{h(0, t)}{1-\epsilon}\left(b_{j}+|\kappa| c_{j}+\frac{B_{0}}{b} d_{j}\right) .
\end{gathered}
$$

Therefore, an upper bound on the truncation error of approximating $u(t)$ using $j$ terms of the infinite series can be found, and it is linear in the coefficients $b_{j}, c_{j}$, and $d_{j}$.

2) Numerical Simulation: For the numerical simulation, we consider incrementing the flow by $1 \mathrm{~m}^{3} / \mathrm{s}$ from its nominal flow $Q_{0}=2.5 \mathrm{~m}^{3} / \mathrm{s}$ in 1 hour $(T=3600 \mathrm{~s})$. We take $\sigma=2$ which implies $y(t)$ to be a Gevrey-function of order 1.5 thus satisfying the convergence condition in Section III-B. The model parameters are $L=1000 \mathrm{~m}, C_{0}=20 \mathrm{~m} / \mathrm{s}, D_{0}=1800 \mathrm{~m}^{2} / \mathrm{s}, B_{0}=7$ $\mathrm{m}$, and $b=1 \mathrm{~m}^{2} / \mathrm{s}$. The infinite series of the control input $u(t)$ is approximated using $j$ terms. The value of $j$ is determined by evaluating the $L_{2}$ norm of the truncation error as a function of $j$ which is given by

$$
\left\|e_{j}\right\|=\left(\int_{0}^{T_{\operatorname{sim}}}\left|e_{j}(t)\right|^{2} d \tau\right)^{1 / 2}
$$

where $T_{\operatorname{sim}}$ is the simulation time. We compute the $L_{2}$ norm of the upper bound error

$$
\begin{array}{r}
\left\|e_{j}\right\| \leq \frac{\sqrt{2}}{2(1-\epsilon)} \frac{\beta}{\alpha} e^{\left(-\frac{\alpha^{2}}{\beta^{2}} T_{\mathrm{sim}}-\alpha L\right)} \sqrt{e^{2 \frac{\alpha^{2}}{\beta^{2}} T_{\mathrm{sim}}}-1} \\
\left(b_{j}+|\kappa| c_{j}+\frac{B_{0}}{b} d_{j}\right)
\end{array}
$$

Fig. 3 shows a comparison between the $L_{2}$ norms of the upper bound computed by (32) and the real error computed by (29) until numerical convergence (the residual goes to machine accuracy for 76 terms). We notice that our upper bound is conservative, (the real error may be two orders of magnitude smaller).

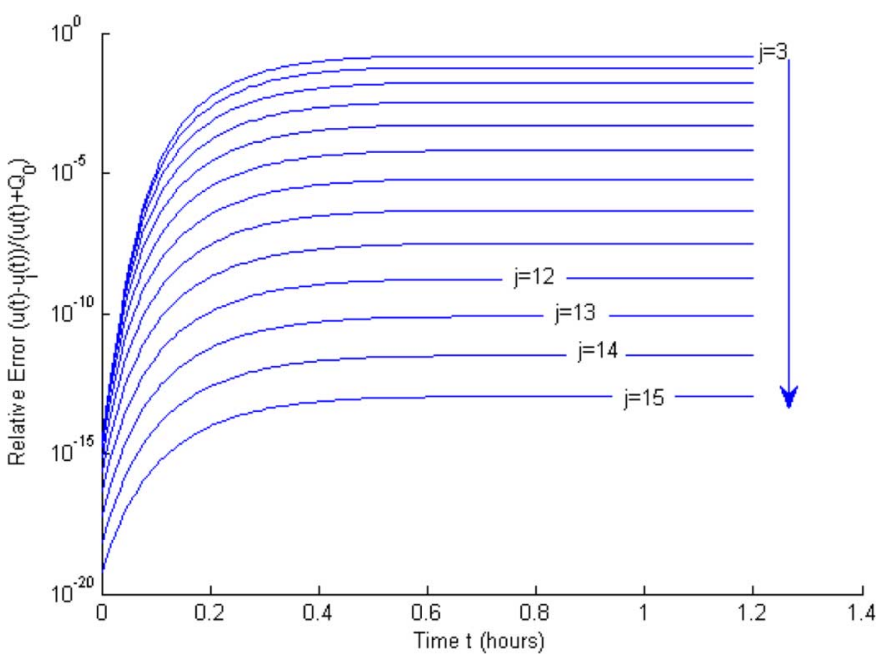

Fig. 4. Effect of adding more terms on the relative error $e_{\text {rel }}(t)=$ $\left|u(t)-u_{j}(t) / Q_{0}+u(t)\right|$ for consecutive values of $j$ starting from $j=3$ to $j=15$.

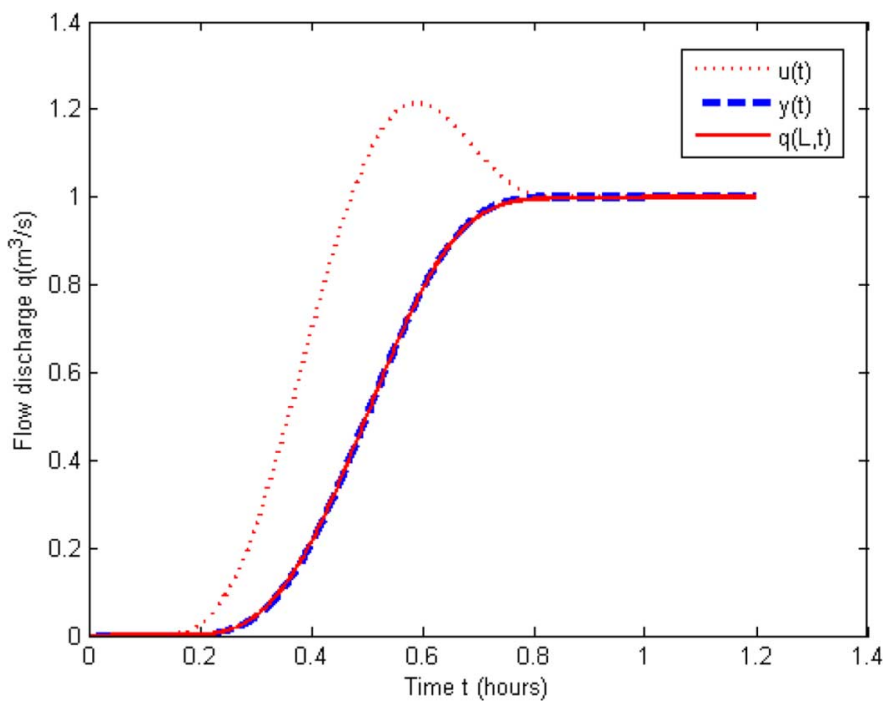

Fig. 5. Results of the numerical simulation of feed-forward control of the Hayami equation. The desired downstream discharge is $y(t)$, the upstream discharge is $u(t)$, and the downstream discharge computed by solving the Hayami model with $b=1 \mathrm{~m}^{2} / \mathrm{s}$ is $q(L, t)$.

Nonetheless, it gives a sufficient condition useful for computational purposes. Fig. 4 shows the effect of adding more terms on the relative error $e_{\text {rel }}(t)=\left|\left(u(t)-u_{j}(t)\right) /\left(Q_{0}+u(t)\right)\right|$. We choose $j=10$ which yields an error of $\left\|e_{j}\right\| \sim 10^{-3}$, and solve (7), (8), (9), and (10) using the Crank-Nicholson scheme. The numerical solution at $x=L$ or $q(L, t)$ is compared to $y(t)$, the desired downstream discharge flow. The results of this simulation are shown in Fig. 5.

The discharge at the downstream follows the desired discharge accurately which validates our control input. We can now compare our result to other problems from the literature.

3) Comparison With the Heat Equation: In the context of thermal systems [17], an explicit open-loop controller was derived for the heat equation with zero gradient boundary conditions. With some simple transformations in time and space we 


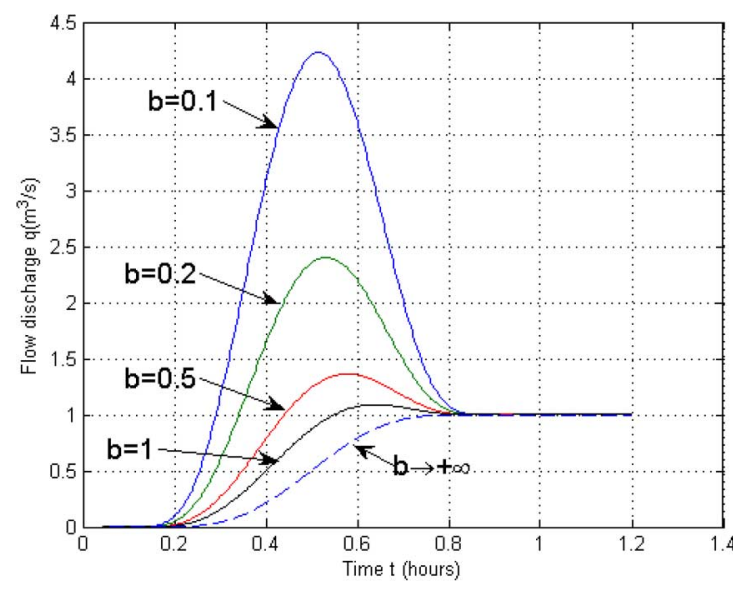

Fig. 6. Effect of varying $b\left(\mathrm{~m}^{2} / \mathrm{s}\right)$ on the upstream discharge or control input $u(t)$.

can relate the results [27] to our problem. The transformed version of the equations of [17] has the following form:

$$
\begin{aligned}
& \forall x \in] 0, L[\forall t \in] 0, T] \quad D_{0} q_{x x}-C_{0} q_{x}=q_{t} \\
& \forall t \in] 0, T] \quad q_{x}(L, t)=0 \\
& \forall x \in] 0, L[\quad q(x, 0)=0 \\
& \forall t \in] 0, T] \quad y(t)=q(L, t) \\
& \forall t \in] 0, T] \quad u(t)=q(0, t) .
\end{aligned}
$$

The solution of the control input for this particular problem is

$$
u_{\text {heat }}(t)=h(0, t)\left(T_{1}(0, t)-\alpha T_{2}(0, t)\right) \text {. }
$$

We can vary the value of the variable $b$ in (27), and observe its effect on $u(t)$. This physically corresponds to changing the height or the width of the weir located at the downstream end of the canal. Fig. 6 shows the effect of varying $b$ on the control input $u$.

We can see that by increasing the value of $b$, the function of $u(t)$ numerically converges to $u_{\text {heat }}(t)$ described by (35). This can be seen directly by inspection of the limit of (27) as $b$ tends to $+\infty$ which would result in (35). Substituting $\kappa=$ $\left(B_{0} / b\right)\left(\alpha^{2} / \beta^{2}\right)-\alpha$ into (27), we obtain

where

$$
u(t)=u_{\text {heat }}(t)+u_{b}(t)
$$

$$
u_{b}(t)=h(0, t) \frac{B_{0}}{b}\left(\frac{\alpha^{2}}{\beta^{2}} T_{2}(0, t)-T_{3}(0, t)\right) .
$$

As $b$ tends to $+\infty$, the boundary effect becomes negligible, and (27) converges in the limit to (35), i.e., in the limit $u$ and $u_{\text {heat }}$ are identical. If we were to use the controller in (35) to control our problem with $b=1 \mathrm{~m}^{2} / \mathrm{s}$, we would obtain the results shown in Fig. 7. The effect can be seen in the transition which takes approximately $1.6 \mathrm{~h}$ instead of $1 \mathrm{~h}$. This shows the considerable importance of boundary conditions on the dynamics of the flow transfer. It is therefore very important to take into account the appropriate physical boundary conditions in the

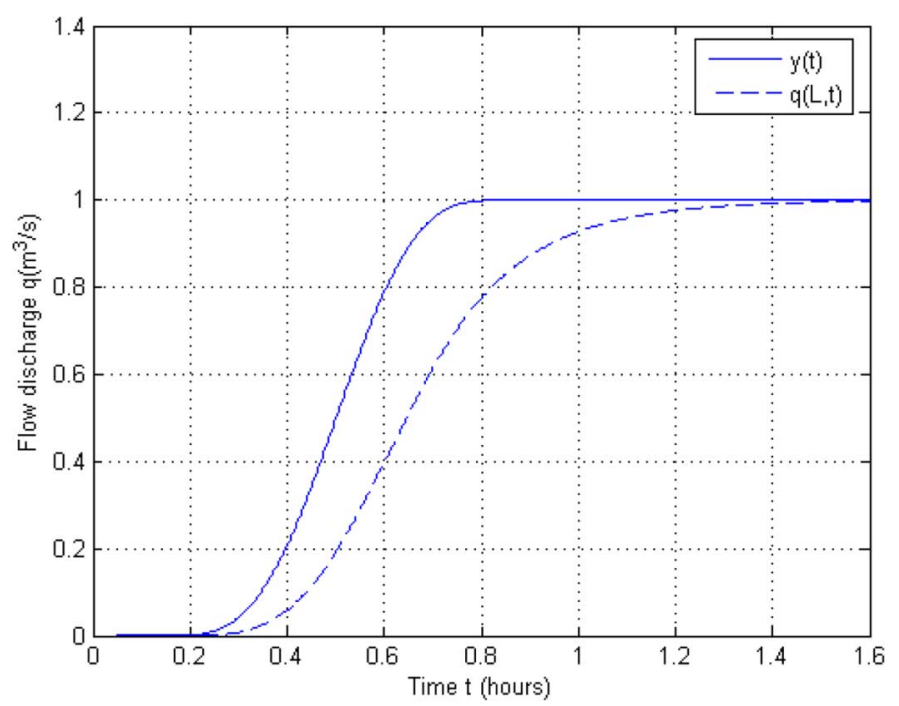

Fig. 7. Consequence of neglecting the boundary conditions in calculating the upstream discharge. The desired downstream discharge is $y(t)$, and the downstream discharge calculated by solving the Hayami model with $b=1 \mathrm{~m}^{2} / \mathrm{s}$ and control input of (35) is $q(L, t)$.

open-loop control design to ensure a scheduled water distribution.

\section{B. Saint-Venant Model Simulation}

In numerous cases, controlling the Saint-Venant equations directly is impractical because of the required knowledge for the geometry of the canal and the Saint-Venant parameters defined in Section II-A. For this reason we have used a simplification of the model to arrive to the Hayami equation which requires only two parameters, $C_{0}$ and $D_{0}$. The coefficient $b$, which represents the downstream boundary condition, can easily be inferred from the weir equation. In this section we show numerically that a calibrated Hayami model would provide us with an open-loop control law that steers the Saint-Venant equation solution at $x=L$ or the flow discharge at the weir to the desired discharge accurately. For the purpose of the simulation we use SIC, a computer program developed by Cemagref [2], [24] to simulate the upstream discharge and the measurement discharge at the downstream. SIC solves the full nonlinear Saint-Venant equations using a finite difference scheme standard in hydraulics (Preissmann scheme). We also study the effect of uncertainties of the Saint-Venant equation parameters on the open-loop control system performance.

1) Hayami Model Identification: The purpose of model identification is to identify the parameters $C_{0}, D_{0}$, and $b$ corresponding to the Hayami model and its boundary condition parameter that would best approximate the real flow governed by the Saint-Venant equations. This is done with an upstream discharge in a form of a step input, the flow discharges are monitored at the upstream and downstream positions. The hydraulic identification is done classically by finding the values of $C_{0}, D_{0}$, and $b$ that minimize the error between the computed downstream discharge by the solution of the Crank-Nicholson scheme [34] and the measured one. We therefore have to solve the following optimization problem: 


$$
\min _{C_{0}, D_{0}, b>0} \int_{0}^{T_{\mathrm{sim}}}\left|q_{\mathrm{SIC}}(\tau)-q_{\mathrm{CN}}\left(C_{0}, D_{0}, b, \tau\right)\right|^{2} d \tau
$$

where $q_{\mathrm{SIC}}$ is the downstream flow generated by SIC, and $q_{\mathrm{CN}}$ is the downstream flow generated by the Crank-Nicholson scheme, $T_{\text {sim }}$ is the simulation time usually larger than the period needed to reach steady state. The nonlinear optimization problem was solved by the MATLAB nonlinear least-square curve fitting function (lsqnonlin). The identification was done using Saint-Venant equations generated data. In our case, the identification was performed around a steady flow regime of $0.4 \mathrm{~m}^{3} / \mathrm{s}$, canal of length $L=4887 \mathrm{~m}$, and bed width $B_{0}=$ $2 \mathrm{~m}$. The average bottom slope is $3.8 \times 10^{-4}$, the Manning coefficient is $0.0213 \mathrm{~m}^{-1 / 3} \mathrm{~s}$, and the weir discharge coefficient is 0.35 . This leads to the following parameters: $C_{0}=0.88 \mathrm{~m} / \mathrm{s}$, $D_{0}=660.19 \mathrm{~m}^{2} / \mathrm{s}$, and $b=0.16 \mathrm{~m}^{2} / \mathrm{s}$. Identification of coefficients of the Hayami equation is standard in hydraulics, and has shown to work well in practice [18].

2) Saint-Venant Control: The experimental canal we would like to simulate has the same properties as the one we have used for identification in the previous section. We are interested in raising the flow at the downstream from $0.4 \mathrm{~m}^{3} / \mathrm{s}$ to $0.5 \mathrm{~m}^{3} / \mathrm{s}$ in $5 \mathrm{~h}$. Setting the variables in Section IV-A to $q_{1}=0.1 \mathrm{~m}^{3} / \mathrm{s}, T=$ $5 \mathrm{~h}$, and $\sigma=1.1$ will define the downstream profile $y(t)$. The control input or the discharge at the upstream can be calculated and the results are shown in Fig. 8. We notice that the open-loop control designed with the Hayami model performs very well on the full nonlinear Saint-Venant equations. As can be seen in Fig. 8, the reference output and the actual output achieved by the Hayami controller on the full Saint-Venant equations are visually almost identical, which confirms the practicality of the method for implementation on canals. This shows that the Hayami model is practical for the design of open-loop control when the corresponding parameters are identified. We extended our results by evaluating the uncertainties on the system parameters, and studying their effect on the performance of the open-loop control system.

3) Sensitivity Analysis: We study the effect of parameter uncertainties in the full nonlinear Saint-Venant model on the downstream discharge. We compute the control input using nominal values of Saint-Venant equations parameters and simulate it with models which incorporate some uncertainties. We specifically study the effect of the Manning and discharge coefficients uncertainties. We experiment with $\pm 20 \%$ variations on the nominal values and compare the downstream discharges of each scenario.

We use the experimental canal described in Section IV-B2 with nominal Manning coefficient $n=0.0213 \mathrm{~m}^{-1 / 3} \mathrm{~s}$, and weir discharge coefficient $C_{w}=0.35$. The control input of Section IV-B2 is simulated under four different scenarios which define the $\pm 20 \%$ variations on the nominal values: Scenario 1: $n=0.0256 \mathrm{~m}^{-1 / 3} s, C_{w}=0.42$, Scenario 2: $n=0.0170 \mathrm{~m}^{-1 / 3} s, C_{w}=0.28$, Scenario 3: $n=0.0256 \mathrm{~m}^{-1 / 3} s, C_{w}=0.28$, and Scenario 4: $n=0.0170 \mathrm{~m}^{-1 / 3} s, C_{w}=0.42$. Fig. 9 shows the result

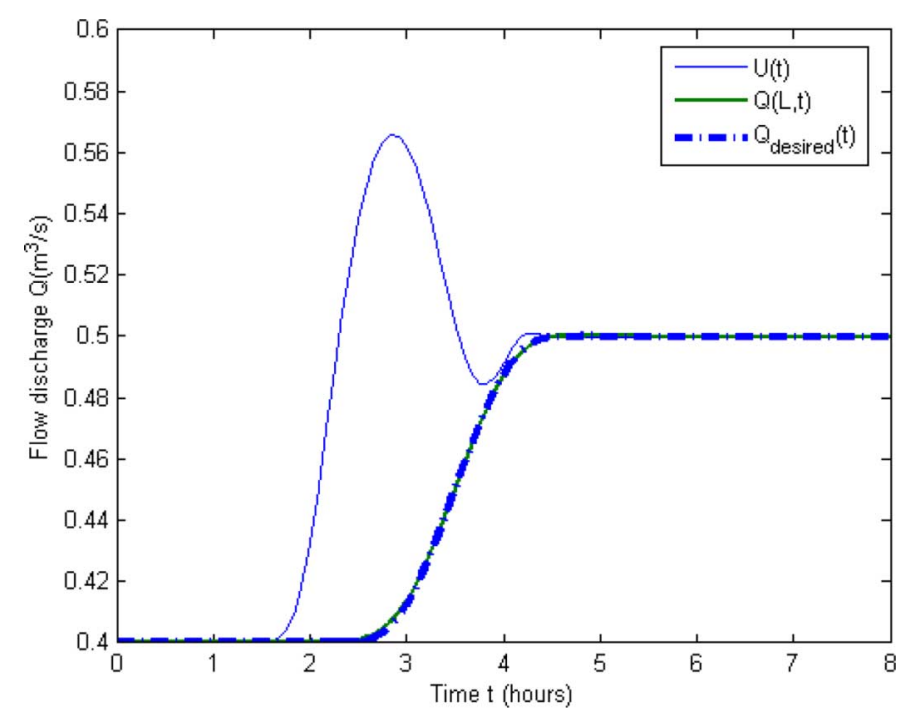

Fig. 8. Results of the implementation of our controller on the full nonlinear Saint-Venant equations. The desired downstream discharge is $Q_{\text {desired }}(t)=$ $Q_{0}+y(t)$, the downstream discharge calculated by solving the Saint-Venant equations in SIC is $Q(L, t)=Q_{0}+q(L, t)$, and the control input of the canal is $U(t)=Q_{0}+u(t)$, where $u(t)$ is calculated using the Hayami model open-loop controller. The nominal flow in the canal is $Q_{0}=0.4 \mathrm{~m}^{3} / \mathrm{s}$.

of the sensitivity analysis. We observe that the dominant effect is due to uncertainties in the Manning coefficient. Underestimating the Manning coefficient as in scenarios 1 and 3, leads to a delay in the downstream discharge delivery (approximately two hours delay to reach the desired downstream discharge). A larger Manning coefficient means more friction and this slows down the upstream discharges to reach the downstream location. In scenarios 2 and 4 (overestimating the Manning coefficient), the downstream discharge reaches its desired value one hour earlier. The peak in the upstream discharge is not fully filtered by the dynamics of the canal and leads to an overshoot in the discharge. The overshoot stabilizes at the desired downstream discharge $\left(0.5 \mathrm{~m}^{3} / \mathrm{s}\right)$ after $2 \mathrm{~h}$.

Overestimating (scenarios 2 and 3), or underestimating (scenarios 1 and 4) the weir discharge coefficient has a very minor, yet opposite effect to uncertainties in the Manning coefficient. Downstream discharges in scenarios 1 and 4 are above the ones in scenarios 3 and 2, respectively. In all cases, the downstream discharge reaches a steady state equal to the desired one with a delay of $2 \mathrm{~h}$.

\section{CONCLUSION}

This brief introduces a new method to design an open-loop control based on the Hayami model for open channel flow control using differential flatness. The controller is obtained as an infinite series (Cauchy-Kovalevskaya decomposition) in terms of the desired downstream discharge flow. We have given sufficient conditions on the downstream profiles to ensure convergence. The effect of the boundary condition is also investigated and compared to previous studies realized for thermal systems. The simulations show satisfactory results for controlling the full Saint-Venant equations. 


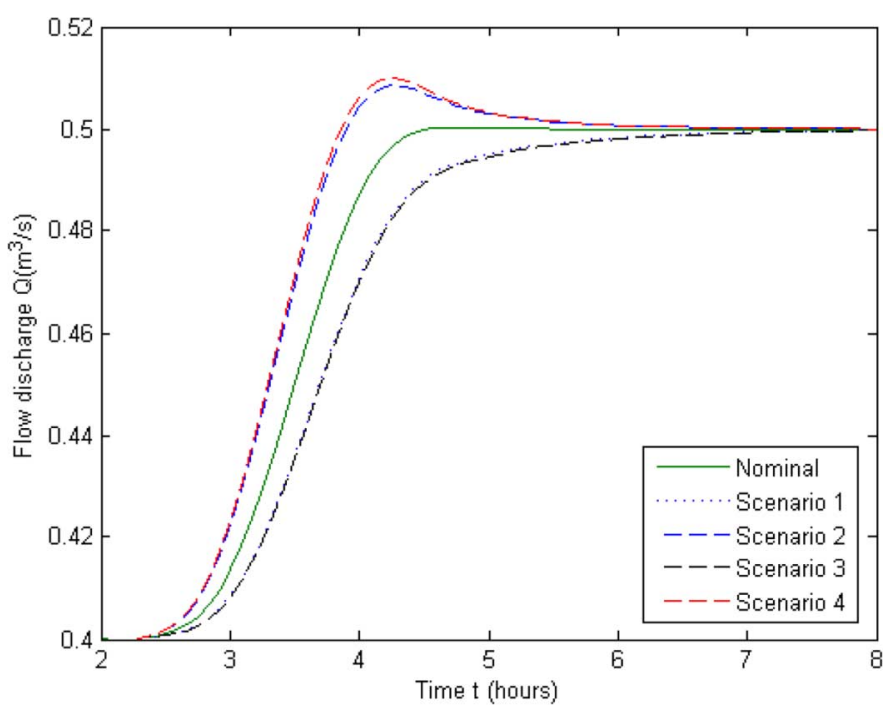

Fig. 9. Simulation results when the Manning and weir discharge coefficients are perturbed around their nominal values $\left(n=0.0213, C_{w}=0.35\right)$. The downstream discharge is computed with four different scenarios. The scenarios correspond to $\pm 20 \%$ uncertainties on the nominal Manning and weir discharge coefficients. Scenario 1: $n=0.0256 \mathrm{~m}^{-1 / 3} \mathrm{~s}, C_{w}=0.42$, Scenario $2: n=$ $0.0170 \mathrm{~m}^{-1 / 3} \mathrm{~s}, C_{w}=0.28$, Scenario $3: n=0.0256 \mathrm{~m}^{-1 / 3} \mathrm{~s}, C_{w}=0.28$, Scenario 4: $n=0.0170 \mathrm{~m}^{-1 / 3} \mathrm{~s}, C_{w}=0.42$.

\section{ACKNOWLEDGMENT}

The authors would like to thank C.-A. Robelin for his initial work on this problem, and conversations which led to these results. N. Petit is gratefully acknowledged for his help in identifying the proper work in flatness which was used in this brief.

\section{REFERENCES}

[1] A. J. C. Barré de Saint-Venant, "Théorie du mouvement non-permanent des eaux avec application aux crues des rivières à l'introduction des marées dans leur lit," Comptes Rendus à l'Académie des Sciences, vol. 73, pp. 148-154, 1871.

[2] J.-P. Baume, P.-O. Malaterre, G. Belaud, and B. L. Guennec, "SIC: A 1D hydrodynamic model for river and irrigation canal modeling and regulation," Métodos Numéricos em Recursos Hidricos, vol. 7, pp. $1-81,2005$.

[3] E. Bautista and A. J. Clemmens, "Response of ASCE task committee test cases to open-loop control measures," J. Irrigation Drainage Eng., vol. 125, no. 4, pp. 179-188, 1999.

[4] E. Bautista, A. J. Clemmens, and T. Strelkoff, "Comparison of numerical procedures for gate stroking," J. Irrigation Drainage Eng., vol. 123, no. 2, pp. 129-136, 1997.

[5] A. Bressan, Hyperbolic Systems of Conservation Laws: The One-Dimensional Cauchy Problem. Oxford, U.K.: Oxford University Press, 2000.

[6] M. Cantoni, E. Weyer, Y. Li, S. K. Ooi, I. Mareels, and M. Ryan, "Control of large-scale irrigation networks," Proc. IEEE, vol. 95, no. 1, pp. 75-91, Jan. 2007.

[7] J. M. Coron, B. D'Andrea-Novel, and G. Bastin, "A Lyapunov approach to control irrigation canals modeled by Saint-Venant equations," presented at the Eur. Control Conf., Karlsruhe, Germany, 1999.
[8] J. de Halleux, C. Prieur, J.-M. Coron, B. de Novel, and G. Bastin, "Boundary feedback control in networks of open-channels," Automatica, vol. 39, pp. 1365-1376, 2003.

[9] F. de Meglio, T. Rabbani, X. Litrico, and A. Bayen, "Feed-forward river flow control using differential flatness," in Proc. 47th IEEE Conf. Decision Control, Cancun, Mexico, 2008, pp. 3903-3910.

[10] W. Dunbar, N. Petit, P. Rouchon, and P. Martin, "Motion planning for a nonlinear stefan problem," ESAIM: Control, Optimisation Calculus Variations, vol. 9, pp. 275-296, 2003.

[11] M. Fliess, J. L. Lévine, P. Martin, and P. Rouchon, "Flatness and defect of non-linear systems: Introductory theory and examples," Int. J. Control, vol. 61, no. 6, pp. 1327-1361, 1995.

[12] M. Fliess, P. Martin, N. Petit, and P. Rouchon, "Active signal restoration for the telegraph equation," in Proc. 38th IEEE Conf. Decision Control, Phoenix, AZ, 1999, vol. 2, pp. 1107-1111.

[13] J. Hadamard, Lectures on Cauchy's Problem in Linear Partial Differential Equations. New York: Courier Dover, 2003.

[14] S. Hayami, "On the Propagation of Flood Waves," Bulletin of the Disaster Prevention Institute, vol. 1, pp. 1-16, 1951.

[15] M. Krstic and A. Smyshlyaev, Boundary Control of PDEs: A Course on Backstepping Designs. Philadelphia, PA: SIAM, 2008.

[16] M. Krstic, "Personal communication," UC Berkeley, Oct. 2007.

[17] B. Laroche, P. Martin, and P. Rouchon, "Motion planning for a class of partial differential equations with boundary control," in Proc. 37th IEEE Conf. Decision Control, Tampa, FL, 1998, vol. 3, pp. 3494-3497.

[18] X. Litrico, "Nonlinear diffusive wave modeling and identification of open channels," J. Hydraulic Eng., vol. 127, no. 4, pp. 313-320, 2001.

[19] X. Litrico and V. Fromion, "Frequency modeling of open channel flow," J. Hydraulic Eng., vol. 130, no. 8, pp. 806-815, 2004.

[20] X. Litrico and V. Fromion, " $H_{\infty}$ control of an irrigation canal pool with a mixed control politics," IEEE Trans. Control Syst. Technol., vol. 14, no. 1, pp. 99-111, Jan. 2006

[21] X. Litrico, V. Fromion, and G. Scorletti, "Robust feedforward boundary control of hyperbolic conservation laws," Netw. Heterogeneous Media, vol. 2, no. 4, pp. 715-729, 2007.

[22] X. Litrico and D. Georges, "Robust continuous-time and discrete-time flow control of a dam-river system: (I) modelling," Appl. Math. Model., vol. 23, no. 11, pp. 809-827, 1999.

[23] A. F. Lynch and J. Rudolph, "Flatness-based boundary control of a nonlinear parabolic equation modelling a tubular reactor," in Nonlinear Control in the Year 2000, London, U.K., 2000, vol. 259, Lecture Notes in Control and Information Sciences, pp. 45-54.

[24] P.-O. Malaterre, "SIC 4.20, simulation of irrigation canals," 2006. [Online]. Available: http://canari.free.fr/sic/sicgb.htm

[25] R. Moussa and C. Bocquillon, "Criteria for the choice of flood-routing methods in natural channels," J. Hydrology, vol. 186, pp. 1-30, 1996.

[26] N. Petit, Y. Creff, P. Rouchon, and P. Cas-Ensmp, "Motion planning for two classes of nonlinear systems with delays depending on the control," in Proc. 37th IEEE Conf. Decision Control, Tampa, FL, 1998, vol. 1, pp. 1007-1011.

[27] N. Petit and P. Rouchon, "Flatness of heavy chain systems," SIAM J. Control Optimization, vol. 40, no. 2, pp. 475-495, 2001.

[28] N. Petit and P. Rouchon, "Dynamics and solutions to some control problems for water-tank systems," IEEE Trans. Autom. Control, vol. 47, no. 4, pp. 594-609, Apr. 2002.

[29] A. de Polyanin, Handbook of Linear Partial Differential Equations for Engineers and Scientists. London, U.K.: Chapman \& Hall/CRC, 2002.

[30] L. Rodino, Linear Partial Differential Operators in Gevrey Spaces. River Edge, NJ: World Scientific, 1993.

[31] J. Rudolph, Sciences et Technologies de l'Automatique "Planning trajectories for a class of linear partial differential equations: An introduction," 2004. [Online]. Available: http://www.esta.see.asso.fr

[32] B. F. Sanders and N. de Katopodes, "Adjoint sensitivity analysis for shallow-water wave control," J. Eng. Mechan., vol. 126, no. 9, pp. 909-919, 2000.

[33] T. Sturm, Open Channel Hydraulics. New York: McGraw-Hill Science Engineering, 2001.

[34] A. Tveito and R. Winther, Introduction to Partial Differential Equations: A Computational Approach. Berlin, Germany: Springer, 1998. 